\title{
Observations of low and intermediate-frequency-peaked BL Lacs above 100 GeV with VERITAS
}

\author{
M. Errando ${ }^{1, a}$ for the VERITAS Collaboration \\ ${ }^{1}$ Department of Physics and Astronomy, Barnard College, Columbia University, NY 10027, USA
}

\begin{abstract}
Most of the $\sim 50$ blazars detected to date at $\mathrm{TeV}$ energies $(E>0.1 \mathrm{TeV})$ are high-frequencypeaked BL Lacs (HBLs). Only a handful episodic detections of low- and intermediate-frequency-peaked BL Lacs (LBL/IBLs, with synchrotron peak frequencies in the infrared and optical regime) have been reported by ground-based gamma-ray telescopes, typically during high-flux states. The VERITAS array located in southern Arizona has observed five known TeV LBL/IBLs since 2009: 3C 66A, W Comae, PKS 1424+240, S5 0716+714 and BL Lacertae, with exposures of 5-10 hours/year, which so far resulted in the detection of a bright, sub-hour timescale gamma-ray flare of BL Lacertae in June 2011. We also report the detection and characterization of two new IBLs: VER J0521+211 and B2 1215+30.
\end{abstract}

\section{Introduction}

The population of $\mathrm{TeV}$ blazars $^{2}$ is dominated by highfrequency-peaked BL Lacs (HBLs), conforming 75\% of the total TeV blazar count [41 out of 55, 1]. HBLs show a maximum of their synchrotron emission in the $\mathrm{X}$-ray regime, and display a low Compton dominance $R_{c}=$ $L_{H E} / L_{s y} \lesssim 1$, defined as the ratio between the luminosity of the high-energy component of the spectral energy distribution and that of the synchrotron emission. At parsec scales, radio imaging of TeV HBLs shows relatively weak jets with only stationary or sub-luminal components [2].

In recent years, the improved sensitivity of the current generation of ground-based Cherenkov telescopes (VERITAS, MAGIC, and H.E.S.S.) revealed TeV emission from a handful of low- and intermediate-frequency-peaked BL Lacs [LBLs/IBLs, 3-11, see Tab. 1]. LBLs and IBLs show different spectral properties than HBLs, with synchrotron emission peaking in the infrared/optical regime

\begin{tabular}{|c|c|c|c|c|}
\hline & RA & Dec & $z$ & ref. \\
\hline $3 \mathrm{C} 66 \mathrm{~A}$ & 022241 & +430235 & $0.41 ?$ & {$[5,12]$} \\
\hline VER J0521+211 & 052155 & +211124 & 0.108 & [11] \\
\hline S5 $0716+714$ & 072153 & +712036 & $0.31 ?$ & [6] \\
\hline B2 $1215+30$ & 121752 & +300701 & $0.13 ?$ & {$[10,13]$} \\
\hline W Comae & 122131 & +281359 & 0.102 & {$[4,14]$} \\
\hline PKS $1424+240$ & 142700 & +234740 & $>0.6$ & [8] \\
\hline AP Librae & 151741 & -242219 & 0.049 & [7] \\
\hline MAGIC J2001+435 & 200113 & +435302 & $?$ & [9] \\
\hline BL Lacertae & 220243 & +421640 & 0.069 & {$[3,15]$} \\
\hline
\end{tabular}

Table 1. TeV-detected IBLs and LBLs.

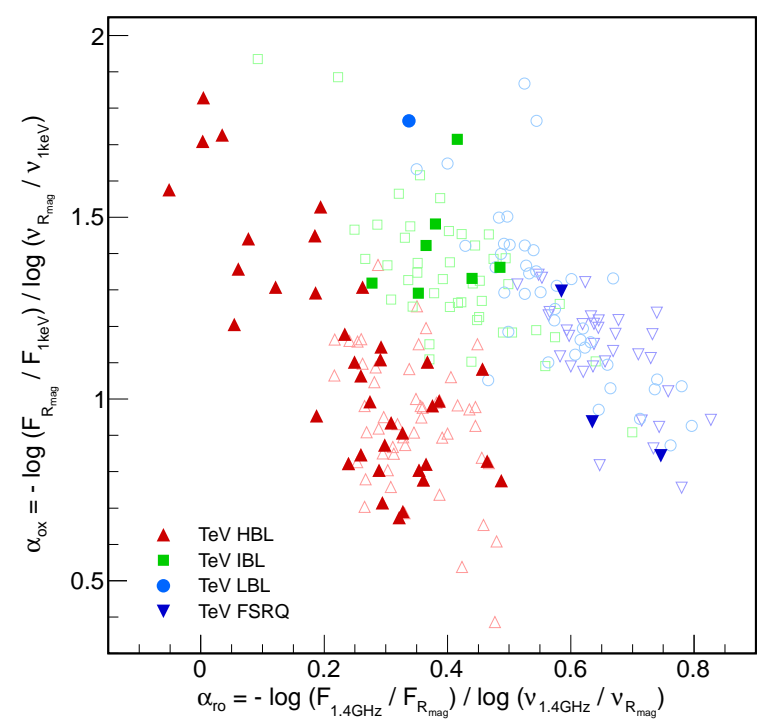

Figure 1. Spectral slopes of the synchrotron component for gamma-ray blazars. The effective spectral indices $\alpha_{\text {ro }}$ and $\alpha_{\text {ox }}$ are defined in the usual way between $1.4 \mathrm{GHz}, 6590 \AA$ and $1 \mathrm{keV}$. Adapted from [11].

and falling towards the X-ray band (Fig. 1). In general, LBLs and IBLs are more powerful, more luminous, and have a richer jet environment compared to HBLs. Accretion rate is thought to be the main driver of these differences $[16,17]$. In this scheme, LBLs accrete close to the Eddington rate, producing powerful and luminous jets, and an optically thick accretion disk whose radiation ionizes the broad line region (BLR) and is reflected by the dust torus, generating sources of external low-energy photons for Compton scattering. On the other hand, HBLs accrete 
at highly sub-Eddington rates, in a radiatively inefficient regime, producing weaker jets that propagate in a 'cleaner' environment. IBLs may represent an intermediate case in terms of their accretion properties, although jet alignment has also been suggested as an explanation for their lower peak frequencies [16]. In the lowest frequency band, a recent compilation of $15 \mathrm{GHz}$ radio images [18] found superluminal components in $3 \mathrm{C} 66 \mathrm{~A}$, S5 0716+714, W Comae, AP Librae, and BL Lacertae. For comparison, only one TeV HBL showed superluminal motion in the same study.

These proceedings report on observations of LBLs and IBLs with VERITAS in the gamma ray band $(E>$ $0.1 \mathrm{TeV}$ ), focusing the recent detections of two new intermediate BL Lacs (VER J0521+211 and B2 1215+30) and a bright $\mathrm{TeV}$ flare from BL Lacertae. More details on these results have been provided in references [11, 13, 15]. The gamma-ray flux of the Crab Nebula is often referred as a reference unit throughout the text, being $1 \mathrm{Crab}=$ $2.1 \times 10^{-10} \mathrm{~cm}^{-2} \mathrm{~s}^{-1}$ above $0.2 \mathrm{TeV}$ [19].

\section{Discovery of TeV emission from VER J0521+211}

VER J0521+211 was suggested as a TeV candidate during a search for clusters of $>30 \mathrm{GeV}$ photons in the Fermi-LAT data. Observations with VERITAS in October 2009 resulted in a significant detection at energies above $0.2 \mathrm{TeV}$ in less than four hours of observation [11], implying a relatively bright $\mathrm{TeV}$ flux at a level of $F_{>0.2 \mathrm{TeV}} \sim$ 0.1 Crab. Follow-up observations revealed a bright flare reaching $\sim 0.3 \mathrm{Crab}$ with a flux doubling time of $\sim 1$ day. Incidentally, VER J0521+211 lies only $3^{\circ}$ away from the Crab Nebula, the first-detected $\mathrm{TeV}$ emitter and the brightest and best-studied gamma-ray source in the sky. The Whipple 10m telescope, and later the VERITAS array, had been observing the Crab Nebula and the region around it for more than two decades, just missing VER J0521+211 outside the edge of their field of view.

The TeV emission detected with VERITAS was spatially associated with RGB J0521.8+2112, an unidentified radio and X-ray source. A complete multiwavelength campaign triggered after the VERITAS detection unambiguously classified VER J0521+211 as a BL Lac-type blazar. The evidence included a one-sided jet resolved in $15 \mathrm{GHz}$ VLBA images, polarized optical emission; pointlike, variable X-ray and gamma-ray emission (Fig. 2), and a featureless optical spectrum. Later observations revealed optical absorption lines, indicating a redshift of $z=0.108$ [20]. Radio imaging also shows electric polarization vectors perpendicular to the jet ridgeline, indicative of optically thin synchrotron emission associated to a wellordered magnetic field aligned in the direction of the jet axis.

The broadband synchrotron emission spectrum from VER J0521+211 shows a peak in the optical band, suggesting a classification as an IBL. However, during the Xray and TeV flare in MJD 55162 (Fig. 2) its synchrotron properties resembled those of TeV HBLs, mainly due to

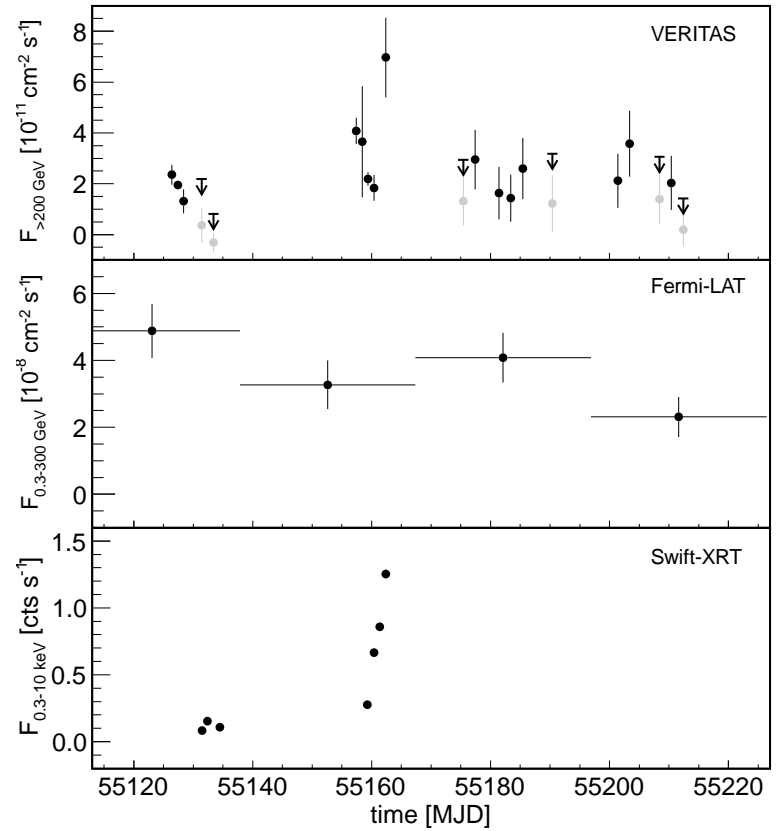

Figure 2. VERITAS $(E>0.2 \mathrm{TeV})$, Fermi-LAT $(0.1-300 \mathrm{GeV})$, and Swift-XRT (0.3-10 keV) light curves of VER J0521+211. Photon fluxes are calculated in 1-day bins for VERITAS and Swift-XRT, and 29.5 days for Fermi-LAT. The VERITAS light curve shows significant flux points (black dots) when the signal exceeds $2 \sigma$, and $95 \%$ confidence level upper limits (black arrows) together with flux points (gray dots) for marginal detections. The error bars on the Swift-XRT rates are at the $\sim 2-7 \%$ level, and are not visible in the plot. Taken from [11].

a hardening of the X-ray spectrum during the high-flux state. Even if it did not figure in TeV-candidate catalogs before the launch of Fermi due to its low Galactic latitude, VER J0521+211 ranks among the brightest blazars known at $\mathrm{TeV}$ energies. At a distance of $z=0.108$, the gammaray luminosity of VER J0521+211 exceeds $2 \times 10^{44} \mathrm{erg} \mathrm{s}^{-1}$, significantly brighter than classical northern blazars such as Mrk 421, Mrk 501 and 1ES 1959+650. Future multiwavelength observations of VER J0521+211 will help to extend the detailed spectral modeling available for nearby HBLs to a more luminous non-HBL blazar.

\section{$3 \mathrm{TeV}$ detection and characterization of B2 1215+30}

B2 $1215+30$ is one of the five sources that were used to define the class of BL Lac-type objects, together with OJ 287, W Comae, AP Librae, and BL Lacertae [21]. Its distance is uncertain, although spectroscopic redshifts of $z=0.130$ and $z=0.237$ can be found in the literature.

$\mathrm{TeV}$ emission from B2 $1215+30$ was reported by MAGIC after observations triggered during an optical high state [10]. The source is only $0.76^{\circ}$ away from 1ES 1218+304, a well-studied TeV HBL that has been extensively monitored with VERITAS since 2009. VERITAS collected a gamma-ray signal from B2 $1215+30$ at $8.9 \sigma$ level after $82 \mathrm{hr}$ of exposure-corrected observations 

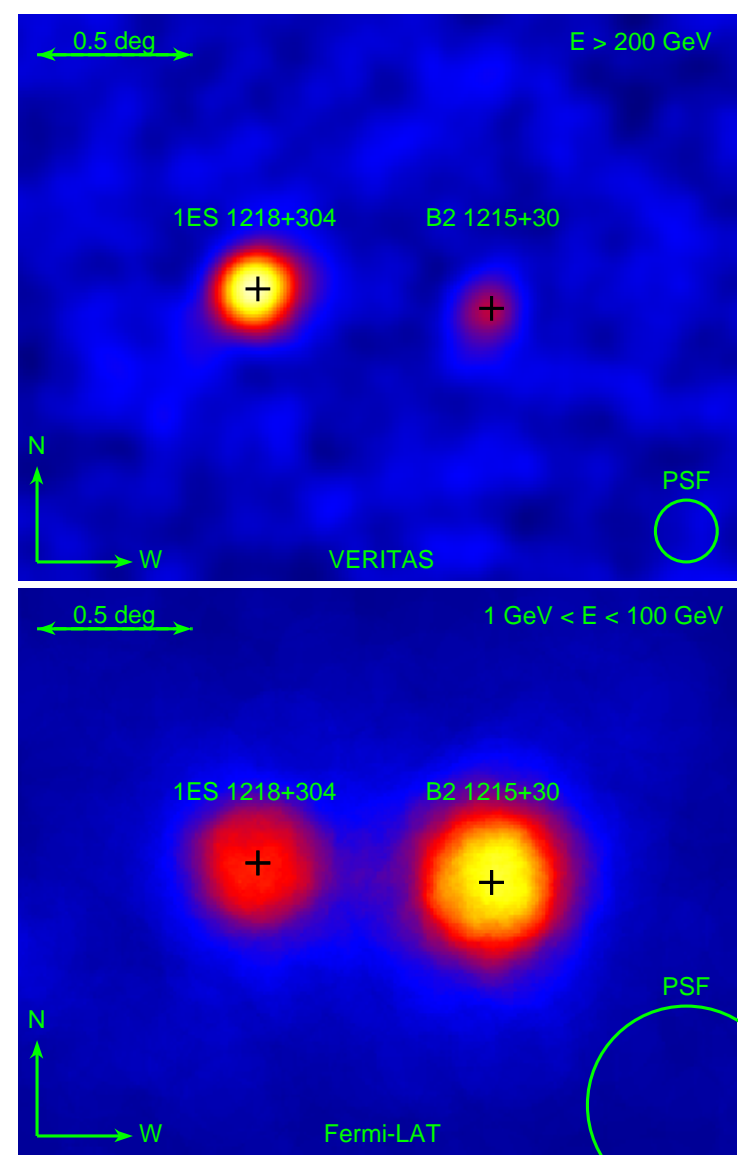

Figure 3. Sky map of the gamma-ray emission measured towards the direction of B2 $1215+30$ (IBL) in two different bands. The top panel (adapted from [13]) shows the signal above $0.2 \mathrm{TeV}$ as measured with VERITAS in 2011. The bottom panel shows a counts map selecting front-converting photons with $E>1 \mathrm{GeV}$ in the Fermi-LAT data (Aug 2008 - Sep 2013). Green circle indicate the $68 \%$ containment angle of both detectors, and the radio location of B2 1215+30 and 1ES 1218+304 is marked with black crosses.

(most of them targeting 1ES 1218+304) spanning over three yearly observing seasons. 1ES $1218+304$ is a bright HBL $\left(F_{>0.2 \mathrm{TeV}} \sim 0.06 \mathrm{Crab}\right)$ showing a power-law spectrum in the $\mathrm{TeV}$ band with $\Gamma_{1218+30}=3.1 \pm 0.3$ [22], while the measured spectral index for B2 $1215+30$ is significantly softer $\left(\Gamma_{1215+30}=3.6 \pm 0.4\right)$. The spectral differences can be visualized in Figure 3, showing how FermiLAT and ground-based gamma-ray telescopes have different sensitivities to distinct source classes. B2 1215+30, with $F_{>0.2 \mathrm{TeV}} \sim 0.03 \mathrm{Crab}$, is significantly weaker than 1ES $1218+304$ in the $\mathrm{TeV}$ band, but the picture reverses at lower energies, where Fermi-LAT measures a much brighter photon flux from B2 $1215+30$.

The gamma-ray signal from B2 $1215+30$ recorded by VERITAS shows clear flux variability in time scales of several months, with a prolonged high state in 2011, in agreement with [10]. Significant variability in shorter time scales could not be resolved. Quasi-simultaneous observations to the 2011 VERITAS detection in the optical band (Super-LOTIS, MDM, Swift-UVOT), X-ray (Swift-XRT), and gamma rays (Fermi-LAT), were used to construct a complete spectral energy distribution, displaying a synchrotron peak in the ultraviolet regime and therefore confirming its classification as an IBL. The multivavelength data was successfully described with a synchrotron selfCompton model with parameters similar to those of other TeV-detected blazars.

\section{TeV flare of BL Lacertae}

The eponymous blazar BL Lacertae was detected by VERITAS during a short-lived TeV flare on 2011 June 28 [15]. The gamma-ray flux over the 35 min of observations was $F_{>0.2 \mathrm{TeV}} \sim 0.3 \mathrm{Crab}$, ten times brighter than in the original discovery publication in 2007 [3]. Figure 4 shows the measured light curve resolved down to 4-min time bins, where intra night variability is first observed for this object in the gamma-ray band. The measured flux has a decay time $t_{v a r}=13 \pm 4 \mathrm{~min}$, and the peak flux reaches $\sim 1.6 \mathrm{Crab}$.

Causality implies that the observed variability timescale is related to the size of the gammaray emission region by $R \lesssim c t_{v a r} \delta(1+z)^{-1} \sim$ $\left(t_{\text {var }} / 13 \mathrm{~min}\right)(\delta / 10) \cdot 7 \times 10^{-5} \mathrm{pc}$, where $\delta$ is the Doppler factor of the emitting plasma. Correlation of the gammaray flaring event with the emergence of a superluminal knot in radio images (also reported in the discovery $\mathrm{TeV}$ emission from BL Lacertae in 2005-6 [23]) has been interpreted as indication of the gamma-ray dissipation region to be at parsec scales from the central black hole. Under that scenario, the compact size of the emitting region of $R \lesssim 7 \times 10^{-5}$ pc would represent only a small fraction of the jet cross section at parsec scales, which would be $\sim 0.1 \mathrm{pc}$ assuming a conical collimated jet. That would suggest that a small emitting region downstream of the jet can be responsible for most of its radiative output.

The broadband gamma-ray spectrum during the flare shows a sharp break between $\Gamma_{\mathrm{GeV}}=1.6 \pm 0.4$ measured by Fermi -LAT and $\Gamma_{\mathrm{TeV}}=3.8 \pm 0.3$ seen by VERITAS (Fig. 4). A spectral break at $\sim 15 \mathrm{GeV}$ would be expected due to Klein-Nishina suppression of the electron-photon cross section if the dominant component of the gamma-ray emission is inverse-Compton scattering of external photons from the BLR [24], as indicated in previous multiwavelength studies of the source both in quiescent and flaring states [25, 26]. Additional steepening of the gammaray spectrum could be due to pair production by gammarays on a dense photon field with frequencies similar to those of BLR photons [27].

A coherent picture is therefore difficult to put forth, as BLR photons are not expected to be dominant at parsec scales but closer to the central black hole. Further observations of BL Lacertae, and of TeV LBLs and IBLs in general are needed to elucidate the location of the gammaray emitting region and the role of external photons in the gamma-ray production and absorption, as well as how the energy dissipation processes change between quiescent states and bright flaring events.

VERITAS is supported by grants from the U.S. Department of Energy Office of Science, the U.S. National Science Foun- 

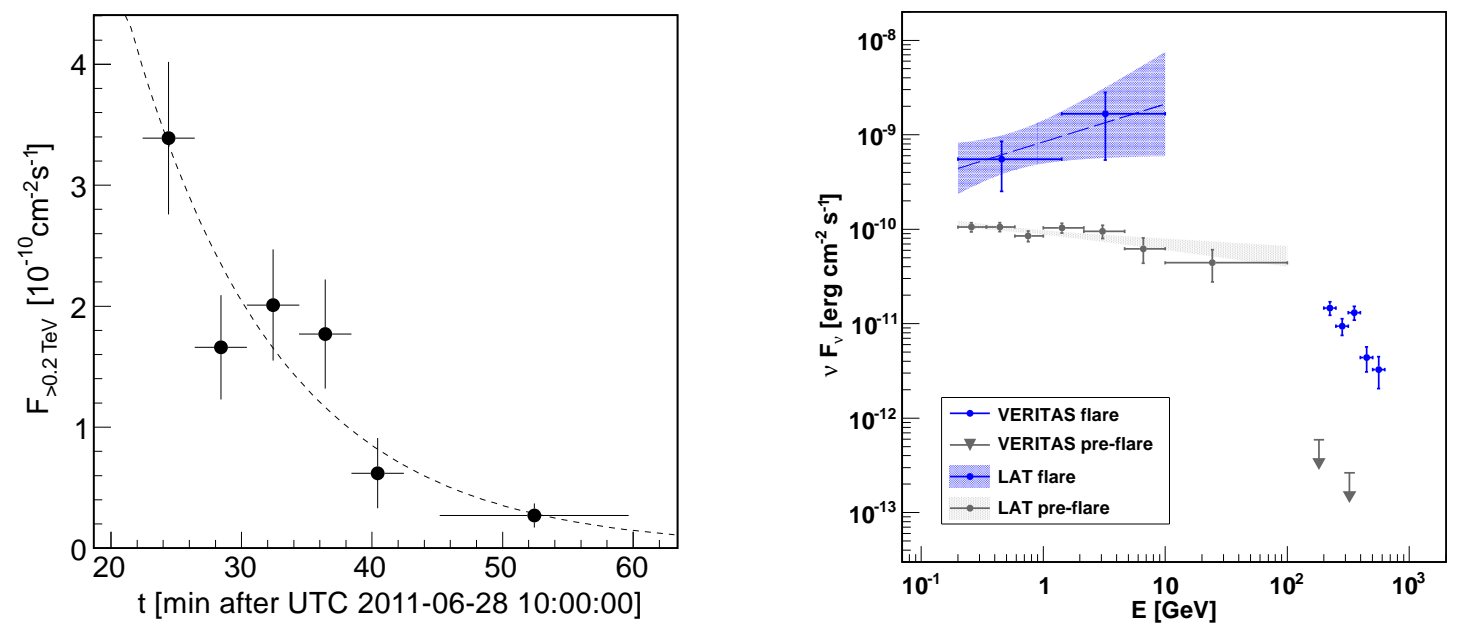

Figure 4. (left) Gamma-ray flux from BL Lacertae measured by VERITAS in 2011 June 28. Flux is binned in intervals as short as $4 \mathrm{~min}$, and the dashed line indicates the best-fit to an exponential flux decay yielding to a $t_{v a r}=13 \pm 4$ min. (right) Broadband gamma-ray spectrum of BL Lacertae from simultaneous Fermi-LAT and VERITAS observations in 2011 June 28. Figures adapted from [15].

dation and the Smithsonian Institution, by NSERC in Canada, by Science Foundation Ireland (SFI 10/RFP/AST2748) and by STFC in the U.K. We acknowledge the excellent work of the technical support staff at the Fred Lawrence Whipple Observatory and at the collaborating institutions in the construction and operation of the instrument.

\section{References}

[1] Wakely, S. P., \& Horan, D. 2008, in Proc. ICRC, 3, 1341

[2] Piner, B. G., Pant, N., \& Edwards, P. G. 2010, ApJ, 723,1150

[3] Albert, J., Aliu, E., Anderhub, H., et al. 2007, ApJL, 666, L17

[4] Acciari, V. A., Aliu, E., Beilicke, M., et al. 2008, ApJL, 684, L73

[5] Acciari, V. A., Aliu, E., Arlen, T., et al. 2009, ApJL, 693, L104

[6] Anderhub, H., Antonelli, L. A., Antoranz, P., et al. 2009, ApJL, 704, L129

[7] Fortin, P., Fegan, S., Horan, D., et al. 2010, in Proc. 25th Texas Symp. Rel. Astrophys., PoS, 123, 199

[8] Acciari, V. A., Aliu, E., Arlen, T., et al. 2010, ApJL, 708, L100

[9] Berger, K., Giavitto, G., Lindfors, E., et al. 2011, in Proc. 2011 Fermi Symp. - eConf C110509, arXiv: 1110.6368

[10] Aleksić, J., Alvarez, E. A., Antonelli, L. A., et al. 2012, A\&A, 544, A142

[11] Archambault, S., Arlen, T., Aune, T., et al. 2013, ApJ, 776, 69
[12] Aleksić, J., Antonelli, L. A., Antoranz, P., et al. 2011, ApJ, 726, 58

[13] Aliu, E., Archambault, S., Arlen, T., et al. 2013, ApJ submitted

[14] Acciari, V. A., Aliu, E., Aune, T., et al. 2009, ApJ, 707, 612

[15] Arlen, T., Aune, T., Beilicke, M., et al. 2013, ApJ, 762,92

[16] Meyer, E. T., Fossati, G., Georganopoulos, M., \& Lister, M. L. 2011, ApJ, 740, 98

[17] Giommi, P., Padovani, P., Polenta, G., et al. 2012, MNRAS, 420, 2899

[18] Lister, M. L., Aller, M. F., Aller, H. D., et al. 2013, AJ in press, arXiv:1308.2713

[19] Hillas, A. M. 1985, in Proc. 19th Int. Cosmic Ray Conf. (La Jolla), NASA Conf. Publ. 3, 445

[20] Shaw, M. S., Romani, R. W., Cotter, G., et al. 2013, ApJ, 764, 135

[21] Strittmatter, P. A., Serkowski, K., Carswell, R., et al. 1972, ApJL, 175, L7

[22] Acciari, V. A., Aliu, E., Arlen, T., et al. 2009, ApJ, 695,1370

[23] Marscher, A. P., Jorstad, S. G., D'Arcangelo, F. D., et al. 2008, Nature, 452, 966

[24] Ghisellini, G., \& Tavecchio, F. 2009, MNRAS, 397, 985

[25] Madejski, G. M., Sikora, M., Jaffe, T., et al. 1999, ApJ, 521, 145

[26] Abdo, A. A., Ackermann, M., Ajello, M., et al. 2011, ApJ, 730, 101

[27] Poutanen, J., \& Stern, B. 2010, ApJL, 717, L118 